\section{A Systematic Review of Literature on Child Sexual Abuse in Saudi Arabia}

\author{
Aref Alsehaimi ${ }^{1,2}$ \\ 1 University of Dundee, UK \\ 2 University of Hail, Saudi Arabia
}

Corresponding author: Aref Alsehaimi

\section{Abstract}

The sexual abuse of children is a serious and persistent problem in Saudi Arabia, as it is in every country, worldwide. However, it is only recently that any kind of official statistics and academic studies have begun to be published on this issue in Saudi Arabia and there remains a great scarceness of data on the subject owing to its sensitivity in a society that is highly conservative and reluctant to discuss such matters openly. Services and programs aiming at addressing the problem in the Kingdom remain at an infant stage, and much still needs to be done in the form of raising public awareness of the issue and setting up mechanisms by which children at risk can readily and safely inform the appropriate authorities of their circumstances and receive prompt attention and protection.

Research, over many years, has shown that any form of childhood abuse is likely to have significant short-, medium-, and long-term consequences for the individual, in terms of their psychological, physical, social, moral, and educational development. Thus, swift action and intervention where abuse has, or is likely to, take place is of great importance. However, the establishment of effective services and programs depends on a thorough understanding of the scale and nature of the problem in the particular social and cultural setting in which it occurs.

The present work consists of a systematic review of the limited literature currently available on child sexual abuse in Saudi Arabia. It examines the findings of the studies carried out to date, including such statistical data on sexual abuse as is currently available, and makes recommendations for future action by researchers and government agencies within the country.

Keywords: Saudi Arabia; Child abuse; Sexual abuse
University of Dundee, Nethergate, Dundee DD1 4HN, United Kingdom.

Tel: 447463334456

Citation: Alsehaimi A. A Systematic Review of Literature on Child Sexual Abuse in Saudi Arabia. J Child Dev Disord. 2016, 2:1.

\section{$\risingdotseq$ a.alsehaimi@dundee.ac.uk}

\section{Introduction}

Child sexual abuse (CSA) is a serious and persistent global problem that, depending on the definition used and the population studied, affects $2 \%-62 \%$ of females and $3 \%-16 \%$ of males [1]. Victims of CSA have been reported to display a range of abnormal behaviors that vary depending upon their age. Preschool children who have been subject to sexual abuse tend to exhibit withdrawn rather than aggressive behavior; however, by school age, victims may engage in aggressive or inappropriate sexual behavior, combined with a depressive mood and low educational attainment. Adolescent victims of CSA may suffer multiple psychiatric and social disorders, including depression, anxiety, low self-esteem, promiscuity, and suicidal thoughts [2]. The impact of the abuse is dependent on a number of factors, such as the age of the child, the age at which the abuse began, the time elapsed since the last instance of abuse, and the relationship of the perpetrator to the victim [3]. Those who have suffered CSA often report difficulties in having normal sexual relationships in adult life, frigidity, or promiscuity. Many perpetrators of CSA were also victims of this form of abuse during their own childhood [4].

The lack of any unequivocal and internationally-agreed-upon definition of the term child sexual abuse continues to hamper research, treatment, and efforts at advocacy. Early researchers used broad and inclusive definitions of child sexual abuse, which are still often cited today [5]. According some definitions of CSA, the perpetrator must be at least 5 years older than the victim, whereas in other definitions no reference is made to the relative 
ages [6]. A commonly used definition is that of Schecter and Roberge, dating back to 1976, which states that sexual abuse of children is "the involvement of dependent, developmentally immature children and adolescents in sexual activities that they do not fully comprehend, are unable to give informed consent to, and that violate the social taboos of family roles". Another recurring theme in many definitions is that CSA involves the exploitation of a child for the sexual gratification of an adult [7].

The literature commonly refers to three main types of CSA. Noncontact CSA includes a variety of actions that do not involve physical contact but are nevertheless sexual in nature, such as indecent exposure, lewd remarks by an adult to a child, or sexual solicitation. The two categories of contact CSA are: (i) those that do not involve intercourse but do include other forms of sexual contact, such as inappropriate touching or fondling, and (ii) those which involve types of intercourse, including oral, vaginal, and anal [8].

CSA often goes unreported, particularly at the time it occurs, for a number of reasons: shame and embarrassment on the part of the victim, guilt (CSA victim's often believe that they are in some way to blame for their abuse), lack of awareness regarding victim's rights, uncertainty and trepidation about relating the experience to strangers, the need to conceal the event from significant people, and fear of not being believed. This under-reporting makes accurate determination of CSA incidence difficult [9].

\section{Aims}

The central aim of this study is to carry out a comprehensive review of published literature on the subject of child sexual abuse in Saudi Arabia in order to establish what is known about the nature and extent of the problem. The researcher will then use the findings as a basis for making recommendations for further research, more effective practice by social workers, and improving protection against CSA through improved programs and policies. Additional goals of the study are: (1) to establish any changes in reported incidence of CSA in Saudi Arabia; (2) to gauge the extent to which researchers and social workers are now ready to address this problem; and ( 3 ) to identify the programs and policies being adopted by the government to meet the challenge of CSA.

\section{Methodology}

The method employed in this study is a systematic review. This began by determining the selection criteria for inclusion and exclusion based on the central aim of the research, i.e., to establish what is known about the nature and extent of the problem, and secondary aims, including to ascertain what official steps have been taken to address the issue. The selection criteria used by the researcher are shown in Table 1.

The search for publications was confined to 1990-2015, which covers the entire period during which social science researchers and health care professionals have been reporting data on child abuse in Saudi Arabia. Unpublished dissertations and theses were included in the search, but other material excluded unless it appeared in peer-reviewed journals. Key terms used for the search were: "Saudi Arabia", "child abuse", "sexual child abuse", and "child protection". General search engines were used initially as a result of which a number of specific online databases and journal indexes were identified.

Following the application of the inclusion and exclusion criteria, five articles and three reports of the 19 identified were found to be relevant to the present study. This stage of the search also involved an interrogation of unpublished dissertations and theses using four databases: COPAC (covering the UK and US), KFNL (King Fahad National Library), (Hail University Library) and (King AbdulAziz University Library). As a result, five items were uncovered as shown in Table 2, four of which proved to be relevant.

Following the literature search and application of the selection criteria, 8 sources were identified as being relevant to this study. The quality of these sources was assessed by examining their relevance to the main aim of the research, their methodological value, and any effect due to biasing.

\section{Results and Discussion}

Both the culture and religion of Saudi Arabia are highly conservative and, until recently, the open discussion of child sexual abuse was taboo [10]. However, among both public and professionals, there has been growing acknowledgement and recognition of the problem of CSA over the past two decades. In part this has been due to the setting up of child protection centers at major medical facilities, along with the implementation of mandatory reporting and data collection strategies [11]. The Saudi Council of Health

Services have adopted the following definition of CSA: "Exposing a child to any adult sexual activity or behavior, including oral sexual contact, touching, caressing, or penetration of the child by genitals or any body part or instrument, as well as verbal sexual harassment. It also includes exploitation of the child in prostitution, pornography, and exploitation through communication tools for sexual purposes" [12].

The annual reports of the Saudi National Family Safety Registry [12-14], which provide a summary of child abuse statistics as recorded by hospital-based child protection centers, give the number of cases of CSA for the years $2010-2012$ as 47 (15.4\% of all reported abuse cases), 136 (22\% of the total), and $56(21.3 \%$ of the total). Between 2000 and 2008 the total number of CSA cases reported to the Suspected Child Abuse and Neglect (SCAN) team, at King Abdul-Aziz Medical City, was 20 (15\% of all child abuse cases). By international standards, these are extremely low numbers. For example, the number of CSA cases recorded in the United Kingdom (with slightly more than double the population of Saudi Arabia) in the 2013 was approximately 31,000. Clearly, the official statistics available on CSA in Saudi Arabia do not reflect the actual scale of the problem and the very low number of reported cases is, in itself, an indication that much more needs to be done to expose the true extent of child sexual abuse in the country and provide adequate support for victims. Moreover, the paucity of reported cases alone may be sufficient to explain the wide variations from one year to the next and, in particular, the drop by more than a half in reported cases between 2011 and 2012.

This study has revealed a deficiency of published work about child sexual abuse in Saudi Arabia. In the literature that is available, 
Table 1 Selection criteria.

\begin{tabular}{|c|cc|}
\hline Selection criteria & Inclusion criteria & Exclusion criteria \\
\hline Language & English and Arabic & Other \\
\hline Publication date & Published from 1990 onwards & Published prior to 1989 \\
\hline Search in & Journal articles, thesis and & Grey literature \\
\hline & government reports & \\
\hline Conditions of interest & Studies conducted on sexual child & Studies discussed that other types \\
\hline Geographical coverage & abuse in Saudi Arabia & of child abuse but did not include \\
\hline Study design & Saudi Arabia & Other countries \\
\hline
\end{tabular}

Table 2 Papers, theses, and other documents identified from main search.

\begin{tabular}{|c|c|c|}
\hline Title of Research & Author (Year) & Relevant \\
\hline $\begin{array}{l}\text { Adverse childhood experiences, chronic } \\
\text { diseases, and risky health }\end{array}$ & Almuneef, Qayad, Aleissa, and & Yes \\
\hline behaviors in Saudi Arabian adults: A pilot study & Albuhairan (2014) & \\
\hline $\begin{array}{c}\text { Pediatrician knowledge, perception, and } \\
\text { experience on child abuse } \\
\text { and neglect in Saudi Arabia }\end{array}$ & & Yes \\
\hline $\begin{array}{c}\text { Child physical and sexual abuse in Dammam, } \\
\text { Saudi Arabia: A }\end{array}$ & AlMadani, Bamousa, Alsaif, & Yes \\
\hline descriptive case-series analysis study & Kharoshah and Alsowayigh (2012) & \\
\hline $\begin{array}{l}\text { Child abuse and neglect in Saudi Arabia: Journey } \\
\text { of recognition to }\end{array}$ & Al Eissaa and Almuneefa (2014) & Yes \\
\hline \multicolumn{3}{|l|}{$\begin{array}{c}\text { implementation of national prevention } \\
\text { strategies. }\end{array}$} \\
\hline $\begin{array}{l}\text { Knowledge, attitudes, and experience of } \\
\text { dentists living in Saudi }\end{array}$ & Al-Dabaan, Newton, and & No \\
\hline Arabia toward child abuse and neglect. & Asimakopoulou (2014) & \\
\hline The National Family Safety Registry report & Health Ministry (2010) & Yes \\
\hline The National Family Safety Registry report & Health Ministry (2011) & Yes \\
\hline The National Family Safety Registry report & Health Ministry (2012) & Yes \\
\hline
\end{tabular}

the authors often point to a change in attitude toward CSA and a growing realization that intervention and child protection measures needs to be strengthened in this area [11]. However, the data available do not suggest that rapid progress has been made over the past few years toward either reporting or tackling the problem. Further research is needed to establish the magnitude and extent of the sexual abuse phenomenon in Saudi Arabia. Only then will it be possible to develop and effective strategy to counter and prevent it.

Until 2007, Saudi law referred victims of sexual abuse to gynecologists for forensic examination. Since then, the Forensic Department has taken over responsibility for the examination of such cases and a special forensic clinic was established at that time [15].

\section{Recommendation}

Although Saudi Arabia has taken some steps in implementing intervention and protection programs aimed at safeguarding children from abuse, much remains to be done. In particular, the issue of child sexual abuse remains highly sensitive and one that has been slow to be addressed within the country. Very little literature is available on the subject and such statistical data as there is merely reflects the fact that the phenomenon is massively underreported. The author recommends that more specialist facilities be established, staffed by trained social workers and medical personnel, where suspected victims can be assessed and given counseling. At the same time, there needs to be a greater emphasis on increasing public awareness of the problem and on establishing secure means, such as $24 \mathrm{~h}$ hotlines, by which children can report abuse without fear of repercussions from their families or the authorities.

CSA is a worldwide phenomenon about which there is an extensive body of literature. Saudi researchers and social workers are urged to add to this international fund of knowledge through further investigation and reporting of CSA in their own country, thus placing the needs of children at risk above the sensitivities of a conservative culture in which such matters have traditionally been hidden from view.

\section{Conclusion}

There is inadequate information on both the incidence and prevalence of child sexual abuse in Saudi Arabia. The literature that is available, as revealed by the search conducted in this study, amounts to a handful of academic papers, theses, and government reports. These publications acknowledge the existence of the problem and that some steps have been taken to address the needs of children in this area, and also provide some rudimentary statistical data. However, these data, such as those 
provided by the National Family Safety Registry in recent years, simply underscore how few cases have actually come to light at an official level and how much more is needed to expose the full scale of the problem, nationwide, and adequately deal with it. The author has made some general recommendations as to how to proceed, but the initiative now must come at a governmental level. A public awareness program is needed along with the means by which children can securely report abuse, but these steps must coincide with the establishment of new facilities that can provide for the safe assessment and counseling of children who are suspected victims of CSA. 


\section{References}

1 Johnson CF (2004) Child sexual abuse. The Lancet 364: 462-470.

2 Glaser D, Wiseman M (2000) Child sexual abuse. Biol Psychiatry 14: 357.

3 Goodyear BP, Fath A, Myers L (2011) Child sexual abuse. Handbook of Child Sexual Abuse: Identification, Assessment, and Treatment pp: 1-28.

4 Lalor K, McElvaney R (2010) Child sexual abuse, links to later sexual exploitation / high-risk sexual behavior, and prevention/treatment programs. Trauma, Violence, Abuse 11: 159-177.

5 Haugaard JJ (2000) The challenge of defining child sexual abuse. Am Psychol 55: 1036.

6 Arriola KR, Louden T, Doldren MA, Fortenberry RM (2005). A metaanalysis of the relationship of child sexual abuse to HIV risk behavior among women. Child Abuse Negl 29: 725-746.

7 Mrazek PB, Kempe CH (2014) Sexually Abused Children and Their Families. Elsevier (Eds.).

8 Andrews G, Corry J, Slade T, Issakidis C, Swanston H, et al. (2004) Child sexual abuse. In Comparative Quantification of Health Risks:
Global and Regional Burden of Disease Attributable to Selected Major Risk Factors. World Health Organization 2: 1851-940.

9 Lalor K, McElvaney R (2010) Child sexual abuse, links to later sexual exploitation / high 9 risk sexual behavior and prevention / treatment programs. Trauma, Violence, and Abuse 11: 159-177.

10 Almuneef M, Qayad M, Aleissa M, Albuhairan F (2014) Adverse childhood experiences, chronic diseases, and risky health behaviors in Saudi Arabian adults: A pilot study. Child Abuse and Negl 38: 1787-1793.

11 Al Eissa M, Almuneef M (2010) Child abuse and neglect in Saudi Arabia: Journey of recognition to implementation of national prevention strategies. Child Abuse and Negl 34: 28-33.

12 The Annual Report (2010) Hospital-Based Child Maltreatment Registry, Saudi Council of Health Services.

13 The Annual Report (2011) Hospital-Based Child Maltreatment Registry, Saudi Council of Health Services.

14 The Annual Report (2012) Hospital-Based Child Maltreatment Registry, Saudi Council of Health Services.

15 AlMadani O, Bamousa M, Alsaif D, Kharoshah MA, Alsowayigh $K$, et al. (2012) Child physical and sexual abuse in Dammam, Saudi Arabia: A descriptive case-series analysis study. Egyptian Journal of Forensic Sciences 2: 33-37 